\title{
L'enquête sur la psittacose et sa prise en charge dans une volière publique : une approche «Une santé »
}

\author{
Hopkins $\mathrm{J}^{1,2^{\star}}$, Alsop $\mathrm{J}^{3}$, Varga $\mathrm{C}^{3}$, Pasma $\mathrm{T}^{3}$, Jekel $\mathrm{P}^{4}$, Rishi L ${ }^{4}$, Hirji $\mathrm{MM}^{4}$, Filejski $\mathrm{C}^{5}$
}

\section{Résumé}

Contexte : La Chlamydophila psittaci est une bactérie zoonotique causant la chlamydia aviaire chez les oiseaux et la psittacose chez les humains. Les volières publiques sont un environnement propice à une exposition à la fois de travail et récréative à des oiseaux malades.

Objectif : Décrire l'enquête sur la chlamydia aviaire et sa prise en charge dans une volière publique au Canada au moyen de l'approche « Une santé ».

Méthodologie : En juin 2013, un loriquet auparavant en bonne santé est mort. L'autopsie a révélé une aérosacculite fongique et une hépatite, et l'application de la technique RT-PCR a donné un résultat positif pour C. psittaci. Les vétérinaires provinciaux ainsi que les autorités sanitaires locales et provinciales ont examiné l'état de santé des oiseaux et du personnel de la volière.

Résultats : Une approche « Une santé » dans la prévention, la détection et l'intervention a été appliquée aux humains et aux oiseaux potentiellement exposés à $C$. psittaci. La volière a été fermée en attendant l'enquête et les mesures d'intervention. Aucun autre cas de maladie aviaire ou humaine n'a été décelé. Les oiseaux ont été traités aux antibiotiques dans l'éventualité où l'oiseau aurait été infectieux avant sa mort. La volière a été nettoyée et désinfectée adéquatement, puis rouverte au public.

Conclusions : La mortalité liée à C. psittaci provenait probablement d'une infection latente ou d'une souche peu virulente, étant donné l'absence de contagion à d'autres oiseaux ou aux humains. Une approche coordonnée et multiservices recourant au cadre "Une santé » a été utilisée avec succès à l'occasion de la première enquête sur $C$. psittaci dans une volière publique au Canada.

\begin{abstract}
Affiliations
${ }^{1}$ Service de santé publique de Hamilton, Hamilton (Ontario)

${ }^{2}$ Département d'épidémiologie clinique et de biostatistique, Université McMaster, Hamilton (Ontario)
\end{abstract}

${ }^{3}$ Bureau du vétérinaire en chef, Ministère de l'Agriculture, de I'Alimentation et des Affaires rurales, Guelph (Ontario)

${ }^{4}$ Santé publique de la région du Niagara, Thorold (Ontario)

${ }^{5}$ Ministère de la Santé et des Soins de longue durée, Toronto (Ontario)

${ }^{*}$ Correspondance : jessica. hopkins@hamilton.ca

Citation suggérée : Hopkins J, Alsop J, Varga C, Pasma T, Jekel P, Rishi L, et al. L'enquête sur la psittacose et sa prise en charge dans une volière publique : une approche "Une santé ». Relevé des maladies transmissibles au Canada 2016;42:126-30. https://doi.org/10.14745/ccdr.v42i05a02f

\section{Introduction}

Le 20 juin 2013, un loriquet à tête bleue (Trichoglossus haematodus) auparavant en bonne santé est mort dans une volière publique en Ontario, au Canada. Les volières intérieures sont de larges enclos qui permettent aux oiseaux de voler et d'interagir dans un environnement contrôlé. Les volières publiques permettent au public de pénétrer dans I'habitat des oiseaux pour les regarder ou interagir avec eux. En vertu du protocole, toutes les mortalités aviaires font l'objet d'une autopsie et d'une analyse. La carcasse de l'oiseau a été soumise au Laboratoire de santé animale de l'Université de Guelph par le vétérinaire traitant. Les échantillons acheminés pour un test de polymérase en chaîne standard et en temps réel (technique RT-PCR) se sont avérés positifs à Chlamydophila psittaci, ce qui suggère une infection latente ou active.

\section{Contexte}

\section{Chlamydia aviaire et psittacose}

La chlamydia aviaire chez les oiseaux et la psittacose (ornithose) chez les humains sont des infections bactériennes causées par C. psittaci (1-3). Bien que les psittacidés (oiseaux de type perroquet), plus spécialement la calopsitte élégante et la perruche ondulée, soient les plus communément infectés, C. psittacia été détectée chez plus de 460 espèces d'oiseaux $(2,4)$. Les personnes les plus à risque sont les propriétaires d'oiseaux ou ceux qui travaillent avec des oiseaux, toutefois, une infection peut se produire dans le contexte d'une exposition fugitive ou en apparence minimale (1-3). Les oiseaux ayant la maladie sous forme latente ou clinique sont infectieux (les premiers de façon intermittente), et ceux qui ont la maladie clinique présentent des signes de léthargie, de l'anorexie, un plumage hérissé, de la somnolence, des tremblements, une perte de poids, une détresse respiratoire, des écoulements 
oculaires ou nasaux séreux ou mucopurulents, une conjonctivite, de la diarrhée et l'excrétion d'urates verts à jaune-verts $(2,5)$.

Les humains ayant la psittacose présentent généralement des symptômes légers similaires à ceux de la grippe, mais peuvent développer des maladies plus sérieuses comme une pneumonie, une encéphalite ou une myocardite $(1,3)$. La période d'incubation peut être longue : de 3 jours à plusieurs semaines chez les oiseaux, et de 1 à 4 semaines chez les humains $(2,6)$. L'excrétion de souches à virulence élevée chez les oiseaux peut particulièrement favoriser des éclosions $(2,3)$. La combinaison d'une excrétion asymptomatique et d'une longue période d'incubation complique les efforts de prévention des éclosions (2).

Au Canada, puisque la surveillance de la chlamydia aviaire ne se fait pas à l'échelle nationale, les taux d'incidence sont inconnus. En Ontario, de un ou deux cas ont été diagnostiqués chaque année dans les cinq dernières années au Laboratoire de santé animale de Guelph, en Ontario (Varga C. Données non publiées; 2014). La psittacose humaine est une maladie à déclaration obligatoire en Ontario (7). Entre 2003 et 2007, moins de cinq cas ont été signalés annuellement (6).

\section{"Une santé "}

"Une santé » est une approche des maladies qui tient compte des interactions entre les gens, les animaux et l'environnement (8) [figure 1]. L'approche "Une santé » valorise le travail interdisciplinaire, reconnaît la complémentarité des compétences de différentes disciplines (par exemple la médecine vétérinaire, la santé publique, la médecine humaine, la santé environnementale) [9] et elle peut être utilisée en soutien aux efforts de prévention, de détection et d'intervention relatifs aux menaces à la santé publique (10).

\section{Justification et objectif}

Une volière publique peut potentiellement exposer ses travailleurs et le public à des zoonoses comme la psittacose. Un seul cas suffit pour nécessiter des mesures de prévention d'éclosion. Il est indispensable de se doter d'un cadre pour soutenir une approche complète et multiservices. Dans cet article, nous rapportons le premier compte rendu connu d'une enquête intégrée sur la chlamydia et la psittacose aviaire et humaine au Canada, en mettant l'accent sur le recours à une approche "Une santé " pour décrire l'intervention multiservice de l'enquête.

\section{Méthodologie}

L'enquête sur l'éclosion a débuté le 18 septembre 2013, quand les résultats de C. psittaci du loriquet se sont avérés positifs, et a été déclarée close le 6 novembre 2013, 44 jours après que la population aviaire de la volière a obtenu des résultats négatifs pour la bactérie à la suite d'un traitement antibiotique. La date de clôture de l'enquête a été décidée sur le fondement que plus d'une période maximale d'incubation s'était écoulée sans qu'aucun nouveau cas n'ait été détecté chez les oiseaux ou les humains.

Figure 1 : Récapitulatif de l'approche « Une santé » et de ses rôles dans l'enquête sur Chlamydophila psittaci

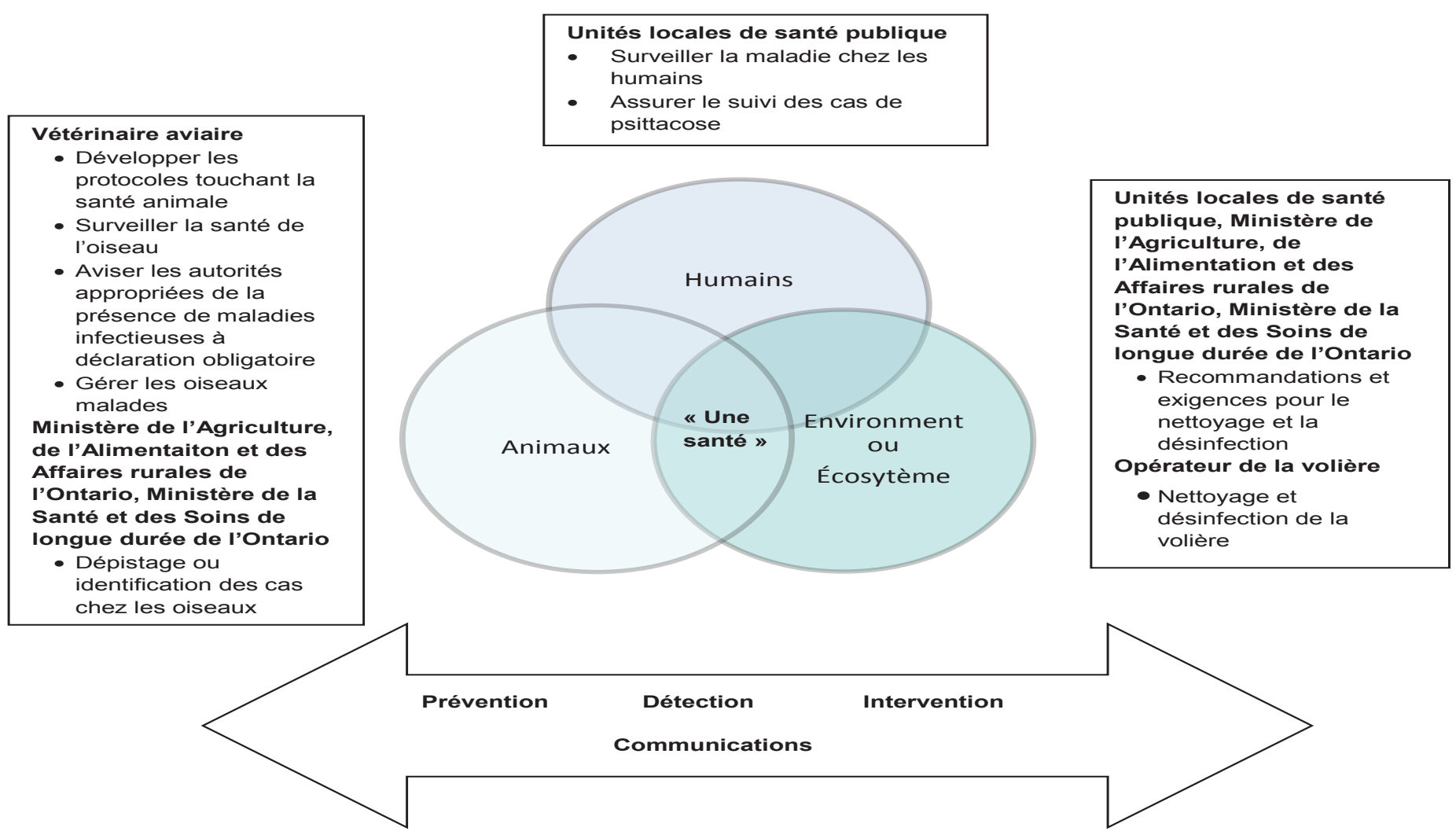




\section{Enquête sur la volière}

La volière était une grande installation ouverte au public. Elle était formée de plusieurs aires divisées par des murs et des portes, chacune dotée d'un système de ventilation séparé. Le loriquet qui est mort appartenait à la volière principale, qui comptait environ 150 oiseaux principalement en liberté. Les loriquets de cette volière principale avaient leur propre cage et n'entraient pas en contact direct avec d'autres oiseaux.

Tel qu'il est exigé par la Loi sur la santé animale (11), le Laboratoire de santé animale a signalé le résultat positif à C. psittaci au ministère de l'Agriculture, de l'Alimentation et des Affaires rurales de l'Ontario, qui a à son tour avisé l'unité locale de santé publique et le ministère de la Santé et des Soins de longue durée de l'Ontario. Les différents organismes ont commencé à travailler avec la volière pour enquêter et limiter la propagation possible de C. psittaci dans les populations aviaire et humaine en utilisant l'approche « Une santé » pour la prévention, la détection et l'intervention.

Afin de détecter $C$. psittaci chez les oiseaux, les vétérinaires du ministère de l'Agriculture, de l'Alimentation et des Affaires rurales de l'Ontario et le vétérinaire de la volière les ont examinés pour détecter des signes de chlamydia. Ils ont colligé des échantillons fécaux et procédé à des écouvillonnages du pharynx chez la population de loriquets, et rassemblé les échantillons fécaux de toutes les autres espèces partageant leur espace aérien. Tous les échantillons ont été testés pour C. psittaci par procédé RT-PCR au Laboratoire de santé animale de Guelph, en Ontario.

\section{Interventions}

Les inspecteurs de santé publique ont évalué l'installation pour déterminer le risque potentiel de psittacose que courraient les visiteurs et le personnel de la volière, et pour déterminer les meilleures mesures pour réduire au minimum ou éliminer complètement les risques de transmission des oiseaux aux humains.

Trois approches principales ont été utilisées : 1) le traitement des oiseaux potentiellement infectés; 2 ) le nettoyage de l'environnement immédiat de la volière pour éliminer les sécrétions d'oiseaux et 3 ) la restriction des contacts humains jusqu'à ce que tous les oiseaux aient été traités.

Les oiseaux de la volière ont été traités à la doxycycline durant 24 jours (le médicament a été introduit dans du nectar pour les loriquets et dans de l'eau pour les autres oiseaux).

Les copeaux de bois ont été retirés des cages et incinérés. Un désinfectant aux propriétés bactéricide, virucide et fongicide a été utilisé pour le nettoyage de l'environnement immédiat des cages à oiseaux et des zones de l'exposition ouvertes au public. Puisqu'il n'existe aucunes directives claires, il y a eu beaucoup de discussions entourant la désinfection de la vie végétale, des sols et des surfaces poreuses et irrégulières. Étant donné que les loriquets étaient en cage et n'entraient en contact direct ni avec le public, ni avec d'autres espèces d'oiseaux, ni avec la vie végétale ou les sols, nous avons choisi de ne pas toucher aux plantes, de désinfecter les surfaces là où c'était possible et d'être à l'affût de tout signe de maladie humaine ou animale.
En vertu de la Loi sur la santé animale (11), un ordre de détention a été délivré pour empêcher la sortie des oiseaux des lieux, et des conditions ont été précisées pour l'incinération de tous les déchets.

Les données de surveillance des symptômes respiratoires chez les humains ont été examinées pour tout signe pouvant renvoyer à C. psittaci. Une évaluation des risques a établi que le personnel de la volière était le plus exposé et présentait le plus grand risque de contracter la psittacose, le public n'ayant de son côté aucun contact direct avec les oiseaux ou leurs cages. L'enquête s'est donc concentrée sur l'état de santé des travailleurs. Le personnel de la volière a été instruit et chargé de surveiller tout symptôme ou signe au cours de la période d'incubation, et d'aviser leur unité locale de santé publique si quoi que ce soit se manifestait.

\section{Résultats}

Le loriquet à tête bleue (Trichoglossus haematodus) qui est mort avait au moins neuf ans, mais son âge exact demeure inconnu. Sa maladie était asymptomatique, et on croyait que le décès était imputable à un traumatisme en raison des ecchymoses observées. Les résultats de la nécropsie (une aérosacculite fongique localisée et une hépatite) ont mené à des examens du foie, de la rate et des reins pour C. psittaci et Coxiella burnetii, et les échantillons se sont avérés positifs pour $C$. psittaci. Aucune autre mortalité aviaire n'avait été signalée dans l'installation au cours de mois précédent l'avis.

La volière principale comptait plus de 150 oiseaux (dont 32 loriquets), dont aucun ne présentait de signe de maladie. Les échantillons rassemblés de matières fécales et du pharynx de tous les loriquets ont donné un résultat négatif lorsqu'ils ont été testés pour C. psittaci par la méthode RT-PCR. Cinq échantillons rassemblés de matières fécales pris d'autres espèces d'oiseaux ont également présenté un résultat négatif lorsqu'ils ont été testés pour C. psittaci. La volière est demeurée fermée pendant huit jours lors du nettoyage et de la désinfection, et un traitement antibiotique de 48 heures a été donné aux oiseaux. L'ordre de détention a été levé au jour 44 , après que les résultats négatifs post-traitement du laboratoire ont été reçus.

\section{Enquête sur les humains}

Les systèmes de surveillance syndromique du service d'urgences n'ont signalé aucune activité suggérant une augmentation de pneumonies ou de syndromes respiratoires au cours de la période observée. Il a été établi que dix travailleurs avaient eu un contact direct soit avec les loriquets, soit avec leur cage et leur contenu. Aucun membre du personnel n'a été malade dans le mois précédant ou suivant la mort du loriquet et, plus précisément, il n'y a eu aucun cas de maladie respiratoire importante. L'unité locale de santé publique a fait parvenir une lettre à tous les employés pour leur demander de rapporter à la santé publique toute maladie assimilable à une psittacose, et de consulter un médecin le cas échéant pour obtenir un dépistage et un traitement adéquats. Aucun cas possible n'a été relevé. 


\section{Approche multiservice coordonnée des échanges}

D'importants échanges ont eu lieu entre les ministères provinciaux (le ministère de l'Agriculture, de l'Alimentation et des Affaires rurales et le ministère de la Santé et des Soins de longue durée), I'unité locale de santé publique, et les opérateurs et le vétérinaire de la volière. Comme il s'agissait du premier cas connu d'une possible éclosion de C. psittaci dans une volière publique intérieure au Canada, nous avons consulté les Centers for Disease Control and Prevention et la National Association of Public Health Veterinarians aux États-Unis pour avoir leur perspective et bénéficier de leur expérience avec des enquêtes similaires. Étant donné l'absence de propagation de C. psittaci et l'absence d'infection active ou latente chez les autres oiseaux, les vétérinaires et les autorités de santé publique se sont entendus sur le fait que C. psittaci n'avait probablement pas été la cause de la mort de l'oiseau et que des mesures appropriées avaient été prises pour réduire au minimum les risques de propagation dans l'éventualité où l'oiseau avait excrété avant sa mort.

\section{Discussion}

À notre connaissance, il s'agit du premier rapport d'une éclosion de chlamydia aviaire dans une volière publique au Canada. Une revue documentaire n'a révélé qu'un petit nombre de rapports d'enquête d'éclosion publiés dans le monde. Matsui et ses collègues (12) ont signalé une éclosion de psittacose liée à un parc aviaire intérieur au Japon qui a entraîné 17 cas chez des humains. Schlossberg et ses collègues (13) ont décrit 13 cas humains de psittacose attribués à une volière située dans le sous-sol d'une église. De petites éclosions faisant suite à des expositions aviaires ont également été signalées, par exemple 18 personnes ayant développé une psittacose après avoir visité une exposition aviaire aux Pays-Bas (14) et 2 cas confirmés (en plus de 46 cas probables et possibles) à la suite d'une foire aviaire en France (15). Des éclosions ont également été signalées dans des volières extérieures, par exemple chez des manchots de Magellan au zoo de San Francisco, sans qu'aucun cas humain soit signalé (16). Ces éclosions ont démontré que des oiseaux qui ne présentent aucun signe clinique peuvent être infectieux. Toutefois, à la différence des éclosions décrites dans les rapports précédents, l'incident sur lequel nous avons enquêté n'a pas débouché sur une éclosion, et ce pour plusieurs raisons possibles : I'oiseau avait très peu d'excrétions ou était infecté par une souche peu virulente; le public n'a pas eu de contact direct avec le loriquet; le système de ventilation du bâtiment était adéquat; des pratiques permettant de maintenir un environnement propre dans la volière étaient déjà en place.

Comme pour toute enquête complexe, plusieurs défis se sont présentés et des questions persistent. Des preuves de la survie de C. psittaci dans l'environnement et du rôle des plantes comme vecteurs passifs manquaient. La volière avait un volume considérable de végétation vivante qui entrait régulièrement en contact avec des fèces et rejets d'oiseaux, mais avec lesquels les visiteurs n'ont pas été en contact. Aussi, les loriquets demeuraient dans leur cage et $n^{\prime}$ entraient pas en contact direct avec la végétation ou le public. Comme il a été mentionné plus haut, nous avons choisi de nous concentrer sur le nettoyage des cages et le remplacement des copeaux dans les cages des loriquets, et nous avons gardé la végétation en optant pour I'hypothèse que son rôle dans la transmission au public serait restreint au minimum étant donné l'absence de contact direct. Nous avons eu la chance qu'aucun autre cas aviaire ou humain se soit présenté. Si tel avait été le cas, il aurait peut-être été plus difficile de distinguer la propagation continue dans la population aviaire du rôle des vecteurs passifs.

Un cycle complet de traitement pour la chlamydia aviaire s'étend généralement sur une période de 30 à 45 jours (5). Étant donné que cette enquête concernait une volière publique, d'importantes pertes se seraient fait sentir si la volière avait été fermée durant toute la durée du traitement. L'aspect économique représente une importante considération dans l'approche "Une santé » et, après avoir examiné la documentation et consulté un expert, nous avons choisi de permettre à la volière de rouvrir après que les oiseaux ont été traités sur 48 heures, étant donné que l'excrétion considérable de l'organisme diminue après cette période (5; communication personnelle. Beaufrère $H$., Collège vétérinaire de l'Ontario; 2013).

Pour terminer, peu importe que le loriquet décédé ait eu une infection active ou latente, en regard de l'incidence des excrétions et du manque d'expérience dans la gestion de la chlamydia aviaire, des ressources ont été allouées à une enquête là où il est probable que l'oiseau mort ait eu une infection latente qui n'aurait jamais mené à une éclosion. De plus amples recherches pour comprendre les facteurs de risque d'éclosion de C. psittaci et des interventions plus efficaces pour prévenir et gérer les cas de C. psittaci aideraient à cibler des mesures de prévention et à allouer les ressources appropriées pour l'intervention.

Cette enquête a été renforcée par la collaboration de multiples juridictions à travers l'approche « Une santé » (figure 1). Les vétérinaires ont détecté une possibilité d'éclosion de psittacose et en ont informé la santé publique. Une réponse coordonnée visant à limiter la propagation de la maladie entre les oiseaux et les humains a été déclenchée et incluait des antibiotiques aux oiseaux ayant une infection latente, des mesures de contrôle de l'infection dans la volière, et une surveillance des cas d'infection chez les oiseaux et les humains. Enfin, des mesures pour prévenir de futures éclosions ont été examinées, y compris l'établissement d'une surveillance constante dans la volière, une biosécurité et des procédures de quarantaine continue pour les oiseaux introduits dans la volière, et un meilleur accès à des désinfectants pour les mains à base d'alcool pour le personnel et les visiteurs de la volière.

En tenant compte tout autant des animaux, des humains et de l'environnement et de l'écosystème en cause, l'approche "Une santé " peut offrir un cadre de réduction des risques dans lequel plusieurs voies d'apparition des risques peuvent être évaluées et gérées, permettant ainsi une intervention de santé publique possiblement plus rapide ou efficace. Cette approche présente toutefois des inconvénients. D'importantes ressources ont été utilisées pour enquêter sur un unique cas de C. psittaci qui était probablement une infection latente présentant un faible potentiel d'éclosion.

Les enquêtes d'éclosions futures et les collaborations réussies tireront profit de propriétaires, d'opérateurs et de vétérinaires 
motivés, qui travaillent avec différentes autorités officielles représentant la santé humaine et animale; d'une compréhension claire des rôles et responsabilités de chacun dans un cadre "Une santé »; de protocoles ou de canaux de communication déjà existants en soutien à la collaboration; d'un accès à une expertise centralisée étant donné la rareté des éclosions de psittacose; de l'intégration de nouvelles preuves scientifiques pour soutenir la prise de décision au fur et à mesure qu'elles deviennent disponibles. Le recours à l'approche "Une santé », bien qu'utile dans cette enquête d'éclosion, tirerait aussi profit d'une analyse au fil de ses utilisations, et possiblement de l'élaboration d'un protocole d'intervention qui pourrait être plus proportionnel au degré de risque évalué.

\section{Remerciements}

Les auteurs remercient le $D^{\text {re }}$ Preeta Kutty pour son rôle de consultant durant l'enquête et ses commentaires utiles sur l'article.

\section{Conflit d'intérêts}

Aucun.

\section{Financement}

Aucun.

\section{Référence}

1. Centers for Disease Control and Prevention. Pneumonia - psittacosis. Atlanta (GA): CDC; http://www.cdc.gov/ pneumonia/atypical/psittacosis.html.

2. Canadian Centre for Occupational Health and Safety. OHS answer fact sheets - psittacosis. Ottawa (ON): CCOHS; c1997-2014. http://www.ccohs.ca/oshanswers/diseases/ psittacosis.html.

3. American Public Health Association. Psittacosis. In: Control of Communicable Diseases Manual. 19th ed. Heymann DL, editor. Washington: American Public Health Association; 2008.
4. Kaleta E, Taday EM. Avian host range of Chlamydophila spp. based on isolation, antigen detection and serology. Avian Pathol. 2003;32:435-61.

5. Smith KA, Campbell CT, Murphy J, Stobierski MG, Tengelsen LA. Compendium of measures to control Chlamydophila psittici infection among humans (psittacosis) and pet birds (avian chlamydiosis), 2010 National Association of State Public Health Veterinarians (NASPHV). J Exotic Pet Med. 2011;20:32-45.

6. Ministry of Health and Long-Term Care. Psittacosis/ ornithosis. In: Ontario Public Health Standards Infectious Diseases Protocol, 2009. Toronto (ON): MOHLTC; 2014. (Disponible en français : http://www.health.gov.on.ca/fr/pro/ programs/publichealth/oph_standards/infdispro.aspx).

7. Health Protection and Promotion Act, R.S.O. 1990, c. H.7. Government of Ontario. (Disponible en français : https:// www.ontario.ca/fr/lois/loi/90h07).

8. Centers for Disease Control and Prevention. About One Health. Atlanta (GA): CDC. http://www.cdc.gov/onehealth/ about.html.

9. One Health Initiative. About the One Health Initiative. http://www.onehealthinitiative.com/about.php.

10. Public Health Agency of Canada. One Health. Ottawa (ON): The Agency; (Disponible en français : http://www.phac-aspc. gc.ca/owoh-umus/index-fra.php).

11. Animal Health Act, 2009, S.O. 2009, Chapter 31. Government of Ontario; (Disponible en français : https:// www.ontario.ca/fr/lois/loi/09a31).

12. Matsui T, Nakashima K, Ohyama T, Kobayashi J, Arima $Y$, Kisimoto T, et al. An outbreak of psittacosis in a bird park in Japan. Epidemiol Infect. 2008;136:492-5.

13. Schlossberg D, Delgado J, Moore MM, Wishner A, Mohn J. An epidemic of avian and human psittacosis. Arch Int Med. 1993;152:2594-6.

14. Koene R, Hautvast J, Zuchner L, Voorn P, RooyackersLemmens $E$, Noel $H$, et al. Local cluster of psittacosis after bird show in the Netherlands, November 2007. Euro Surveill. 2007;12:E071213.1.

15. Belchior E, Barataud D, Ollivier R, Capek I, Laroucau K, de Barbeyrac B, et al. Psittacosis outbreak after participation in a bird fair, Western France, December 2008. Epidemiol Infect. 2008;139:1637-41.

16. Jencek JE, Beaufrère H, Tully TN Jr, Garner MM, Dunker $\mathrm{FH}$, Baszler TV. An outbreak of Chlamydophila psittaci in an outdoor colony of Magellanic penguins (Spheniscus magellanicus). J Avian Med Surg. 2012;26:225-31. 